TAIWANESE JOURNAL OF MATHEMATICS

Vol. 17, No. 1, pp. 221-237, February 2013

DOI: $10.11650 /$ tjm.17.2013.2133

This paper is available online at http://journal.taiwanmathsoc.org.tw

\title{
VARIOUS INEQUALITIES IN REPRODUCING KERNEL HILBERT SPACES
}

\author{
Nguyen Du Vi Nhan and Dinh Thanh Duc
}

Abstract. In this paper, we examine various reproducing kernel Hilbert spaces $\mathcal{H}_{K_{1}}$ and $\mathcal{H}_{K_{2}}$ such that the inequality

$$
\operatorname{det}\left[\left\langle F_{i} G_{i}, F_{j} G_{j}\right\rangle_{\mathcal{H}_{K_{1} K_{2}}}\right]_{i, j=1}^{m} \leq C^{m} \operatorname{det}\left[\left\langle F_{i}, F_{j}\right\rangle_{\mathcal{H}_{K_{1}}}\left\langle G_{i}, G_{j}\right\rangle_{\mathcal{H}_{K_{2}}}\right]_{i, j=1}^{m}
$$

holds for all $F_{j} \in \mathcal{H}_{K_{1}}, G_{j} \in \mathcal{H}_{K_{2}}$, where $m$ is a positive integer, $C$ is a constant which is independent on $F_{j}$ and $G_{j}$ for all $j=1,2, \ldots, m$, and $\mathcal{H}_{K_{1} K_{2}}$ is the Hilbert space admitting the reproducing kernel $K_{1} K_{2}$.

\section{INTRODUCTION}

Let $K_{1}(x, y)$ and $K_{2}(x, y)$ be two positive definite quadratic form functions on $E \times E$ and let $\mathcal{H}_{K_{1}}$ and $\mathcal{H}_{K_{2}}$ be two Hilbert spaces admitting the reproducing kernels $K_{1}$ and $K_{2}$, respectively. By the Schur's theorem we see that the usual product $K(x, y)=$ $K_{1}(x, y) K_{2}(x, y)$ is again a positive definite quadratic form function on $E \times E$. Then, the reproducing kernel Hilbert space $\mathcal{H}_{K}$ admitting the reproducing kernel $K(x, y)$ is the restriction of the tensor product $\mathcal{H}_{K_{1}} \otimes \mathcal{H}_{K_{2}}$ to the diagonal set; that is given by (see $[2,7]$ or $[20]$ for more details)

Proposition 1.1. ([7]). Let $\left\{g_{j}\right\}_{j}$ and $\left\{h_{j}\right\}_{j}$ be some complete orthonormal systems in $\mathcal{H}_{K_{1}}$ and $\mathcal{H}_{K_{2}}$, respectively. Then, the reproducing kernel Hilbert space $\mathcal{H}_{K}$ is comprised of all functions on $E$ which are represented as, in the sense of absolutely convergence on $E$,

$$
f(x)=\sum_{i, j} \alpha_{i, j} g_{i}(x) h_{j}(x) \quad \text { on } \quad E, \quad \sum_{i, j}\left|\alpha_{i, j}\right|^{2}<\infty
$$

Received June 14, 2012, accepted July 2, 2012.

Communicated by Ngai-Ching Wong.

2010 Mathematics Subject Classification: 26D10, 26D15, 46E22, 44A35, 32A10, 26A46.

Key words and phrases: Cauchy-Schwarz inequality, Reproducing kernel Hilbert space, Ronvolution, Holomorphic function, Absolutely continuous function.

This research is funded by Vietnam National Foundation for Science and Technology Development (NAFOSTED) under grant number 101.01-2011.15. 
and its norm in $\mathcal{H}_{K}$ is given by

$$
\|f\|_{\mathcal{H}_{K}}^{2}=\min \left\{\sum_{i, j}\left|\alpha_{i, j}\right|^{2}\right\},
$$

where $\left\{\alpha_{i, j}\right\}$ are considered satisfying (1.1).

In particular, we obtain the inequality

$$
\left\|f_{1} f_{2}\right\|_{\mathcal{H}_{K_{1} K_{2}}(E)} \leq\left\|f_{1}\right\|_{\mathcal{H}_{K_{1}}(E)}\left\|f_{2}\right\|_{\mathcal{H}_{K_{2}}(E)} .
$$

From (1.2), various norm inequalities (see $[7,14,15,16,17,18,19])$ in reproducing kernel Hilbert spaces were obtained, which were generalized and reproved using various technics and were expanded for various directions with applications to inverse problems and partial differential equations (see $[1,4,5,6,8,10,11,9,12,13]$ ).

In this paper, by investigating various reproducing kernel Hilbert spaces $\mathcal{H}_{1}, \mathcal{H}_{2}$, and $\mathcal{H}$ and using the Cauchy-Schwarz inequality, we establish the inequality in the following form

$$
\operatorname{det}\left[\left\langle F_{i} G_{i}, F_{j} G_{j}\right\rangle_{\mathcal{H}}\right]_{i, j=1}^{m} \leq C^{m} \operatorname{det}\left[\left\langle F_{i}, F_{j}\right\rangle_{\mathcal{H}_{1}}\left\langle G_{i}, G_{j}\right\rangle_{\mathcal{H}_{2}}\right]_{i, j=1}^{m},
$$

where $m$ is a positive integer, $F_{j} \in \mathcal{H}_{1}, G_{j} \in \mathcal{H}_{2}$ and $C$ is a constant which is independent on $F_{j}$ and $G_{j}$ for all $j=1,2, \ldots, m$. Note that, the left-hand side of (1.3) is the Gram determinant of the vectors $F_{1} G_{1}, \ldots, F_{n} G_{n}$ on $\mathcal{H}$, while the right-hand side of (1.3) is the determinant of Hadamard product of two Gram matrices associated with the vectors $F_{1}, \ldots, F_{m}$ on $\mathcal{H}_{1}$ and $G_{1}, \ldots, G_{m}$ on $\mathcal{H}_{2}$.

We will see that all the inequalities in this paper of the form (1.3) are best possible, because, for example, for $F_{j} \in \mathcal{H}_{1}$ and $G_{j} \in \mathcal{H}_{2}$ such that

$$
\left\|F_{j} G_{j}\right\|_{\mathcal{H}}^{2}=C\left\|F_{j}\right\|_{\mathcal{H}_{1}}^{2}\left\|G_{j}\right\|_{\mathcal{H}_{2}}^{2}, \quad j=1,2, \ldots, m,
$$

the equality holds in (1.3). Taking profit of the reproducing kernels theory, we can find out the cases holding in the equalities (1.4). See the deep theory of A. Yamada [22]. However, we think for the complicated structures in (1.3) the equality problem is very difficult and it is new challenge.

\section{Spaces of Square Summable Series}

Let $\Psi$ be a weight on $\Delta_{r}=\{z \in \mathbb{C}:|z|<r\}$, that means,

$$
\Psi(z)=\sum_{n=0}^{\infty} \psi(n) z^{n}, \quad \psi_{n}>0, \quad n \geq 0
$$


be holomorphic in $\Delta_{r}$ and having $\Delta_{r}$ as its disk of convergence. Let $K_{\Psi}(w, z)$ be a reproducing kernel on $\Delta_{r}$ defined by the expansion

$$
K_{\Psi}(w, z)=\sum_{n=0}^{\infty} \psi(n) w^{n} \bar{z}^{n} .
$$

Then, the reproducing kernel Hilbert space $\ell_{\Psi}=\mathcal{H}_{K_{\Psi}}$ is composed of all holomorphic functions $F(z)$ defined by

$$
F(z)=\sum_{n=0}^{\infty} f(n) z^{n} \quad \text { on } \Delta_{r}
$$

with finite norms

$$
\|F\|_{\ell_{\Psi}}^{2}=\sum_{n=0}^{\infty} \frac{|f(n)|^{2}}{\psi(n)} .
$$

For two weights $\Psi$ and $\Phi$ on $\Delta_{r}$ with the power series $\Psi(z)=\sum_{n=0}^{\infty} \psi(n) z^{n}$ and $\Phi(z)=\sum_{n=0}^{\infty} \varphi(n) z^{n}$, we have

$$
\Psi(z) \Phi(z)=\sum_{n=0}^{\infty}(\psi * \varphi)(n) z^{n}, \quad z \in \Delta_{r},
$$

where

$$
(\psi * \varphi)(n)=\sum_{k=0}^{n} \psi(k) \varphi(n-k)>0, \quad n \geq 0
$$

and so

$$
K_{\Psi}(w, z) K_{\Phi}(w, z)=K_{\Psi \Phi}(w, z) \quad \text { for } w, z \in \Delta_{r}
$$

Let $F(z)=\sum_{n=0}^{\infty} f(n) z^{n} \in \ell_{\Psi}$ and $G(z)=\sum_{n=0}^{\infty} g(n) z^{n} \in \ell_{\Phi}$. Then, the following inequality (see [21, pp. 121-122] or [11])

$$
\sum_{n=0}^{\infty} \frac{|(f * g)(n)|^{2}}{(\psi * \varphi)(n)} \leq \sum_{n=0}^{\infty} \frac{|f(n)|^{2}}{\psi(n)} \sum_{n=0}^{\infty} \frac{|g(n)|^{2}}{\varphi(n)}
$$

shows that $F G \in \ell_{\Psi \Phi}$ and

$$
\|F G\|_{\ell_{\Psi \Phi}} \leq\|F\|_{\ell_{\Psi}}\|G\|_{\ell_{\Phi}}
$$

Moreover, we have the following theorem.

Theorem 2.1. Let $\Psi$ and $\Phi$ be two weights on $\Delta_{r}$ and $F_{j} \in \ell_{\Psi}, G_{j} \in \ell_{\Phi}$ for $j=1,2, \ldots, m$. Then, we have the following inequality

$$
\operatorname{det}\left[\left\langle F_{i} G_{i}, F_{j} G_{j}\right\rangle_{\ell_{\Psi \Phi}}\right]_{i, j=1}^{m} \leq \operatorname{det}\left[\left\langle F_{i}, F_{j}\right\rangle_{\ell_{\Psi}}\left\langle G_{i}, G_{j}\right\rangle_{\ell_{\Phi}}\right]_{i, j=1}^{m} .
$$


Proof. Suppose that $F_{j}(z)=\sum_{n=0}^{\infty} f_{j}(n) z^{n} \in \ell_{\Psi}$ and $G_{j}(z)=\sum_{n=0}^{\infty} g_{j}(n) z^{n} \in$ $\ell_{\Phi}$ for $j=1,2, \ldots, m$. Then, by the expressions

$$
F_{j}(z) G_{j}(z)=\sum_{n=0}^{\infty}\left(f_{j} * g_{j}\right)(n) z^{n}, \quad j=1,2, \ldots, m,
$$

and by properties of determinants and limiting arguments, we have

$$
\operatorname{det}\left[\left\langle F_{i} G_{i}, F_{j} G_{j}\right\rangle_{\ell_{\Psi \Phi}}\right]_{i, j=1}^{m}=\frac{1}{m !} \sum_{n_{1}=0}^{\infty} \cdots \sum_{n_{m}=0}^{\infty} \frac{\left|\operatorname{det}\left[\left(f_{i} * g_{i}\right)\left(n_{j}\right)\right]_{i, j=1}^{m}\right|^{2}}{\prod_{j=1}^{m}(\psi * \varphi)\left(n_{j}\right)} .
$$

Note that

$$
\operatorname{det}\left[\left(f_{i} * g_{i}\right)\left(n_{j}\right)\right]_{i, j=1}^{m}=\sum_{k_{1}=0}^{n_{1}} \cdots \sum_{k_{m}=0}^{n_{m}} \operatorname{det}\left[f_{i}\left(k_{j}\right) g_{i}\left(n_{j}-k_{j}\right)\right]_{i, j=1}^{m} .
$$

Hence, in view of the Cauchy-Schwarz inequality, we have

$$
\frac{\left|\operatorname{det}\left[\left(f_{i} * g_{i}\right)\left(n_{j}\right)\right]_{i, j=1}^{m}\right|^{2}}{\prod_{j=1}^{m}(\psi * \varphi)\left(n_{j}\right)} \leq \sum_{k_{1}=0}^{n_{1}} \cdots \sum_{k_{m}=0}^{n_{m}} \frac{\left|\operatorname{det}\left[f_{i}\left(k_{j}\right) g_{i}\left(n_{j}-k_{j}\right)\right]_{i, j=1}^{m}\right|^{2}}{\prod_{j=1}^{m} \psi\left(k_{j}\right) \varphi\left(n_{j}-k_{j}\right)} .
$$

Denote by $S_{m}$, the set of all permutations of the set $\{1,2, \ldots, m\}$. The Laplace formula shows that

$$
\begin{aligned}
& \frac{\left|\operatorname{det}\left[f_{i}\left(k_{j}\right) g_{i}\left(n_{j}-k_{j}\right)\right]_{i, j=1}^{m}\right|^{2}}{\prod_{j=1}^{m} \psi\left(k_{j}\right) \varphi\left(n_{j}-k_{j}\right)} \\
= & \frac{\operatorname{det}\left[f_{i}\left(k_{j}\right) g_{i}\left(n_{j}-k_{j}\right)\right]_{i, j=1}^{m} \overline{\operatorname{det}\left[f_{i}\left(k_{j}\right) g_{i}\left(n_{j}-k_{j}\right)\right]_{i, j=1}^{m}}}{\prod_{j=1}^{m} \psi\left(k_{j}\right) \varphi\left(n_{j}-k_{j}\right)} \\
= & \sum_{\sigma \in S_{m}} \sum_{\gamma \in S_{m}} \operatorname{sgn} \sigma \operatorname{sgn} \gamma \prod_{i=1}^{m} \frac{f_{i}\left(k_{\sigma(i)}\right) \overline{f_{i}\left(k_{\gamma(i)}\right)} g_{i}\left(n_{\sigma(i)}-k_{\sigma(i)}\right) \overline{g_{i}\left(n_{\gamma(i)}-k_{\gamma(i)}\right)}}{\psi\left(k_{\sigma(i)}\right) \varphi\left(n_{\sigma(i)}-k_{\sigma(i)}\right)}
\end{aligned}
$$

which is, by letting $\lambda=\gamma^{-1} \circ \sigma$,

$$
\begin{aligned}
& =\sum_{\sigma \in S_{m}} \sum_{\lambda \in S_{m}} \operatorname{sgn} \lambda \prod_{i=1}^{m} \frac{f_{i}\left(k_{\sigma(i)}\right) \overline{f_{\lambda(i)}\left(k_{\sigma(i)}\right)}}{\psi\left(k_{\sigma(i)}\right)} \frac{g_{i}\left(n_{\sigma(i)}-k_{\sigma(i)}\right) \overline{g_{\lambda(i)}\left(n_{\sigma(i)}-k_{\sigma(i)}\right)}}{\varphi\left(n_{\sigma(i)}-k_{\sigma(i)}\right)} \\
& =\sum_{\sigma \in S_{m}} \operatorname{det}\left[\frac{f_{i}\left(k_{\sigma(i)}\right) \overline{f_{j}\left(k_{\sigma(i)}\right)}}{\psi\left(k_{\sigma(i)}\right)} \frac{g_{i}\left(n_{\sigma(i)}-k_{\sigma(i)}\right) \overline{g_{j}\left(n_{\sigma(i)}-k_{\sigma(i)}\right)}}{\varphi\left(n_{\sigma(i)}-k_{\sigma(i)}\right)}\right]_{i, j=1}^{m},
\end{aligned}
$$

and so 


$$
\sum_{k_{1}=0}^{n_{1}} \cdots \sum_{k_{m}=0}^{n_{m}} \frac{\left|\operatorname{det}\left[f_{i}\left(k_{j}\right) g_{i}\left(n_{j}-k_{j}\right)\right]_{i, j=1}^{m}\right|^{2}}{\prod_{j=1}^{m} \psi\left(k_{j}\right) \varphi\left(n_{j}-k_{j}\right)}=\sum_{\sigma \in S_{m}} \operatorname{det}\left[\left(\frac{f_{i} \overline{f_{j}}}{\psi} * \frac{g_{i} \overline{g_{j}}}{\varphi}\right)\left(n_{\sigma(i)}\right)\right]_{i, j=1}^{m} \text {. }
$$

Therefore,

$$
\begin{aligned}
\operatorname{det}\left[\left\langle F_{i} G_{i}, F_{j} G_{j}\right\rangle_{\ell_{\Psi \Phi}}\right]_{i, j=1}^{m} & =\frac{1}{m !} \sum_{n_{1}=0}^{\infty} \cdots \sum_{n_{m}=0}^{\infty} \frac{\left|\operatorname{det}\left[\left(f_{i} * g_{i}\right)\left(n_{j}\right)\right]_{i, j=1}^{m}\right|^{2}}{\prod_{j=1}^{m}(\psi * \varphi)\left(n_{j}\right)} \\
& \leq \frac{1}{m !} \sum_{\sigma \in S_{m}} \sum_{n_{1}=0}^{\infty} \cdots \sum_{n_{m}=0}^{\infty} \operatorname{det}\left[\left(\frac{f_{i} \overline{f_{j}}}{\psi} * \frac{g_{i} \overline{g_{j}}}{\varphi}\right)\left(n_{\sigma(i)}\right)\right]_{i, j=1}^{m} \\
& =\operatorname{det}\left[\left\langle F_{i}, F_{j}\right\rangle_{\ell_{\Psi}}\left\langle G_{i}, G_{j}\right\rangle_{\ell_{\Phi}}\right]_{i, j=1}^{m} .
\end{aligned}
$$

This concludes the proof.

Remark 2.2. The inequality (2.2) is best possible. Indeed, equality in (2.2) implies that equality holds in (2.3). This happens only if equality holds in Hölder's inequality, i.e, only if for $n_{j} \geq 0, j=1,2, \ldots, m$, there exists a number $h\left(n_{1}, \ldots, n_{m}\right) \in \mathbb{C}$ such that

$$
\sum_{k_{1}=0}^{n_{1}} \cdots \sum_{k_{m}=0}^{n_{m}}\left|\frac{\operatorname{det}\left[f_{i}\left(k_{j}\right) g_{i}\left(n_{j}-k_{j}\right)\right]_{i, j=1}^{m}}{\prod_{j=1}^{m} \psi\left(k_{j}\right) \varphi\left(n_{j}-k_{j}\right)}\right|^{2}=h\left(n_{1}, \ldots, n_{m}\right)
$$

for all $k_{j}=0,1, \ldots, n_{j}, j=1,2, \ldots, m$.

It is difficult to determine, in general, under what conditions equality can hold in (2.4). However, we see that if there exist numbers $h_{j}(n) \in \mathbb{C}$ such that

$$
\frac{f_{j}(k) g_{j}(n-k)}{\psi(k) \varphi(n-k)}=h_{j}(n), \quad k=0,1, \ldots, n
$$

for all $j=1,2, \ldots, m$, then (2.4) holds. From (2.5) we derive (see [11])

$$
f_{j}(n)=A_{j} \psi(n){\overline{w_{j}}}^{n}, \quad g_{j}=B_{j} \varphi(n){\overline{w_{j}}}^{n}, \quad n=0,1,2, \ldots
$$

for some $w_{j} \in \Delta_{r}$ and some constants $A_{j}$ and $B_{j}$ for $j=1,2, \ldots, m$. Hence,

$$
F_{j}(z)=A_{j} K_{\Psi}\left(z, w_{j}\right), \quad G_{j}(z)=B_{j} K_{\Phi}\left(z, w_{j}\right), \quad z \in \Delta_{r}
$$

for some $w_{j} \in \Delta_{r}, j=1,2, \ldots, m$.

Notice that for $F_{j}$ and $G_{j}$ satisfying (2.6) we have the equalities

$$
\left\|F_{j} G_{j}\right\|_{\ell_{\Psi \Phi}}=\left\|F_{j}\right\|_{\ell_{\Psi}}\left\|G_{j}\right\|_{\ell_{\Phi}}, \quad j=1,2, \ldots, m .
$$




\section{Applications to Spaces of Holomorphic Functions}

First, let us consider the Fischer space $\mathcal{F}_{A}(A>0)$ comprising all entire functions $F(z)$ with finite norms

$$
\|F\|_{\mathcal{F}_{A}}^{2}:=\frac{A}{\pi} \iint_{\mathbb{C}}|F(z)|^{2} e^{-A|z|^{2}} d x d y .
$$

For $F_{1}, F_{2} \in \mathcal{F}_{A}$, we have (see [5, pp. 350-354])

$$
\left\langle F_{1}, F_{2}\right\rangle_{\mathcal{F}_{A}}=\left\langle F_{1}, F_{2}\right\rangle_{\ell_{\Psi}},
$$

where $\Psi(z)=e^{A z}, z \in \mathbb{C}$.

Let $A>0, B>0, \Psi(z)=e^{A z}$ and $\Phi(z)=e^{B z}$ for $z \in \mathbb{C}$. Then,

$$
\Psi(z) \Phi(z)=e^{(A+B) z}, \quad z \in \mathbb{C} .
$$

Combining (2.1) with (3.1) gives us

$$
\|F G\|_{\mathcal{F}_{A+B}} \leq\|F\|_{\mathcal{F}_{A}}\|G\|_{\mathcal{F}_{B}}
$$

for $F \in \mathcal{F}_{A}$ and $G \in \mathcal{F}_{B}$. A more special case of inequality (3.2) was proved by Saitoh [16].

Then, Theorem 2.1 gives us the following theorem.

Theorem 3.1. Let $A$ and $B$ be two positive real numbers. Then, the following inequality

$$
\operatorname{det}\left[\left\langle F_{i} G_{i}, F_{j} G_{j}\right\rangle_{\mathcal{F}_{A+B}}\right]_{i, j=1}^{m} \leq \operatorname{det}\left[\left\langle F_{i}, F_{j}\right\rangle_{\mathcal{F}_{A}}\left\langle G_{i}, G_{j}\right\rangle_{\mathcal{F}_{B}}\right]_{i, j=1}^{m} .
$$

holds for $F_{j} \in \mathcal{F}_{A}$ and $G_{j} \in \mathcal{F}_{B}$ for $j=1,2, \ldots, m$.

If $F_{j} \in \mathcal{F}_{A}$ and $G_{j} \in \mathcal{F}_{B}$ such that

$$
F_{j}(z)=A_{j} e^{A \overline{w_{j}} z}, \quad G_{j}(z)=B_{j} e^{B \overline{w_{j}} z}, \quad z \in \mathbb{C}
$$

for some $w_{j} \in \mathbb{C}$ and some constants $A_{j}$ and $B_{j}, j=1,2, \ldots, m$, then the equality holds in (3.3).

Now, for $\alpha \geq 1$, we consider the Bergman-Selberg kernels $K_{\alpha}(w, z)$ on the open unit disk

$$
K_{\alpha}(w, z)=\frac{1}{(1-w \bar{z})^{\alpha}} \quad \text { for } w, z \in \Delta_{1} .
$$

Then (see [4, p. 280]), the Hilbert space $\mathcal{H}_{K_{\alpha}}$ coincides with the space of holomorphic functions $F(z)=\sum_{n=0}^{\infty} f(n) z^{n}$ on $\Delta_{1}$ such that

$$
\sum_{n=0}^{\infty} \frac{\Gamma(\alpha) \Gamma(n+1)}{\Gamma(\alpha+n)}|f(n)|^{2}<\infty,
$$


equipped with the inner product

$$
\langle F, G\rangle_{\mathcal{H}_{K_{\alpha}}}=\sum_{n=0}^{\infty} \frac{\Gamma(\alpha) \Gamma(n+1)}{\Gamma(\alpha+n)} f(n) \overline{g(n)},
$$

for $F(z)=\sum_{n=0}^{\infty} f(n) z^{n}$ and $G(z)=\sum_{n=0}^{\infty} g(n) z^{n}$.

When $\alpha>1, \mathcal{A}_{\alpha}=\mathcal{H}_{K_{\alpha}}$ is also a Bergman weighted space on the open unit disk $\Delta_{1}$ with weight $\frac{\alpha-1}{\pi}\left(1-|z|^{2}\right)^{\alpha-2}$, that is, $\mathcal{A}_{\alpha}$ coincides with the space of holomorphic functions $F(z)$ on $\Delta_{1}$ such that

$$
\|F\|_{\mathcal{A}_{\alpha}}^{2}:=\int_{\Delta_{1}}|F(z)|^{2} d \mu_{\alpha}(z)<\infty,
$$

where $\mu_{\alpha}$ is the measure on $\Delta_{1}$ given by

$$
d \mu_{\alpha}(z)=\frac{\alpha-1}{\pi}\left(1-|z|^{2}\right)^{\alpha-2} d x d y, \quad z=x+i y .
$$

For $F_{1}, F_{2} \in \mathcal{A}_{\alpha}$ we obtain

$$
\left\langle F_{1}, F_{2}\right\rangle_{\mathcal{A}_{\alpha}}=\left\langle F_{1}, F_{2}\right\rangle_{\ell_{\Psi}},
$$

where

$$
\Psi(z)=\frac{1}{(1-z)^{\alpha}}, \quad z \in \Delta_{1} .
$$

Hence, for $\alpha>1$ and $\beta>1$, the following inequality

$$
\|F G\|_{\mathcal{A}_{\alpha+\beta}} \leq\|F\|_{\mathcal{A}_{\alpha}}\|G\|_{\mathcal{A}_{\beta}}
$$

holds for all $F \in \mathcal{A}_{\alpha}$ and $G \in \mathcal{A}_{\beta}$. Furthermore, by applying Theorem 2.1, we have

Theorem 3.2. Let $\alpha>1$ and $\beta>1$. Then, the following inequality

$$
\operatorname{det}\left[\left\langle F_{i} G_{i}, F_{j} G_{j}\right\rangle_{\mathcal{A}_{\alpha+\beta}}\right]_{i, j=1}^{m} \leq \operatorname{det}\left[\left\langle F_{i}, F_{j}\right\rangle_{\mathcal{A}_{\alpha}}\left\langle G_{i}, G_{j}\right\rangle_{\mathcal{A}_{\beta}}\right]_{i, j=1}^{m} .
$$

holds for $F_{j} \in \mathcal{A}_{\alpha}$ and $G_{j} \in \mathcal{A}_{\beta}$ for $j=1,2, \ldots, m$.

If $F_{j} \in \mathcal{A}_{\alpha}$ and $G_{j} \in \mathcal{A}_{\beta}$ such that

$$
F_{j}(z)=\frac{A_{j}}{\left(1-\overline{w_{j}} z\right)^{\alpha}}, \quad G_{j}(z)=\frac{B_{j}}{\left(1-\overline{w_{j}} z\right)^{\beta}}, \quad z \in \Delta_{1}
$$

for some $w_{j} \in \Delta_{1}$ and some constants $A_{j}$ and $B_{j}, j=1,2, \ldots, m$, then the equality holds in (3.6).

It remains the case when $\alpha=1$. The function

$$
K_{1}(w, z)=\frac{1}{1-w \bar{z}}, \quad w, z \in \Delta_{1},
$$


is the Szegö reproducing kernel for the Hilbert space $\mathcal{H}=\mathcal{H}_{K_{1}}$ comprising all holomorphic functions $F(z)$ on $\Delta_{1}$ with finite norms

$$
\|F\|_{\mathcal{H}}^{2}=\frac{1}{2 \pi} \int_{\partial \Delta_{1}}|f(z)|^{2}|d z| .
$$

Then, for $F, G \in \mathcal{H}$ we have $F G \in \mathcal{A}_{2}$, and moreover,

$$
\|F G\|_{\mathcal{A}_{2}} \leq\|F\|_{\mathcal{H}}\|G\|_{\mathcal{H}} .
$$

The above inequality was also proved by Saitoh [15]. However, he proved the inequality on a very general domain and furthermore, solved the equality problem for the inequality.

Theorem 3.3. For $F_{j}, G_{j} \in \mathcal{H}, j=1,2, \ldots, m$, we have the following inequality

$$
\operatorname{det}\left[\left\langle F_{i} G_{i}, F_{j} G_{j}\right\rangle_{\mathcal{A}_{2}}\right]_{i, j=1}^{m} \leq \operatorname{det}\left[\left\langle F_{i}, F_{j}\right\rangle_{\mathcal{H}}\left\langle G_{i}, G_{j}\right\rangle_{\mathcal{H}}\right]_{i, j=1}^{m} .
$$

If $F_{j} \in \mathcal{H}$ and $G_{j} \in \mathcal{H}$ such that

$$
F_{j}(z)=\frac{A_{j}}{1-\overline{w_{j}} z}, \quad G_{j}(z)=\frac{B_{j}}{1-\overline{w_{j}} z}, \quad z \in \Delta_{1}
$$

for some $w_{j} \in \Delta_{1}$ and some constants $A_{j}$ and $B_{j}, j=1,2, \ldots, m$, then the equality holds in (3.9).

Finally, note that

$$
\frac{1+w \bar{z}}{1-w \bar{z}}
$$

and

$$
\frac{1+w \bar{z}}{(1-w \bar{z})^{2}}
$$

are the reproducing kernels for the Hilbert spaces $\mathcal{P}$ and $\mathcal{Q}$ comprising all holomorphic functions $F(z)$ on $\Delta_{1}$ with finite norms

$$
\|F\|_{\mathcal{P}}^{2}=\frac{1}{4} \int_{\partial \Delta_{1}}|f(z)|^{2}|d z|+\frac{\pi}{2}|f(0)|^{2},
$$

and

$$
\|F\|_{\mathcal{Q}}^{2}=\frac{1}{2 \pi} \iint_{\Delta_{1}} \frac{|f(z)|^{2}}{|z|} d z
$$

respectively (see $[20$, pp. 66, 69]). Since

$$
\frac{1+w \bar{z}}{(1-w \bar{z})^{2}}=\frac{1+w \bar{z}}{1-w \bar{z}} \cdot \frac{1}{1-w \bar{z}}, \quad z, w \in \Delta_{1},
$$

it follows from (2.1) that for $F \in \mathcal{P}$ and $G \in \mathcal{H}$ we have $F G \in \mathcal{Q}$ and,

$$
\|F G\|_{\mathcal{Q}} \leq\|F\|_{\mathcal{P}}\|G\|_{\mathcal{H}} .
$$


Theorem 3.4. For $F_{j} \in \mathcal{P}, G_{j} \in \mathcal{H}, j=1,2, \ldots, m$, we have the following inequality

$$
\operatorname{det}\left[\left\langle F_{i} G_{i}, F_{j} G_{j}\right\rangle_{\mathcal{Q}}\right]_{i, j=1}^{m} \leq \operatorname{det}\left[\left\langle F_{i}, F_{j}\right\rangle_{\mathcal{P}}\left\langle G_{i}, G_{j}\right\rangle_{\mathcal{H}}\right]_{i, j=1}^{m}
$$

If $F_{j} \in \mathcal{P}$ and $G_{j} \in \mathcal{H}$ such that

$$
F_{j}(z)=A_{j} \frac{1+\overline{w_{j}} z}{1-\overline{w_{j}} z}, \quad G_{j}(z)=\frac{B_{j}}{1-\overline{w_{j}} z}, \quad z \in \Delta_{1}
$$

for some $w_{j} \in \Delta_{1}$ and some constants $A_{j}$ and $B_{j}, j=1,2, \ldots, m$, then the equality holds in (3.12).

\section{Spaces of Square Integrable Functions}

The Hardy space (see [3, pp. 113-114]) $\mathcal{D}_{q}=\mathcal{D}_{q}\left(\mathbb{C}^{+}\right), q>0$, is the space of all functions $F(z)$, holomorphic in the right half plane $\mathbb{C}^{+}=\{z \in \mathbb{C}: \operatorname{Re} z>0\}$, of the form

for functions $f$ satisfying

$$
F(z)=\int_{0}^{\infty} e^{-z t} f(t) d t
$$

$$
\int_{0}^{\infty}|f(t)|^{2} t^{1-2 q} d t<\infty, \quad q>0
$$

$\mathcal{D}_{q}$ is the reproducing kernel Hilbert space, admitting the Hardy reproducing kernel

$$
K_{q}(w, z)=\int_{0}^{\infty} e^{-t(w-\bar{z})} t^{2 q-1} d t=\frac{\Gamma(2 q)}{(w+\bar{z})^{2 q}} \quad \text { on } \mathbb{C}^{+} \times \mathbb{C}^{+},
$$

with the norm

$$
\|F\|_{\mathcal{D}_{q}}^{2}=\int_{0}^{\infty}|f(t)|^{2} t^{1-2 q} d t
$$

In particular (see [20, p. 74]), for $q>\frac{1}{2}, K_{q}(w, z)$ is the Bergman-Selberg reproducing kernel on the half plane $\mathbb{C}^{+}$comprising all holomorphic functions $F(z)$ on $\mathbb{C}^{+}$with finite norms

$$
\|F\|_{\mathcal{D}_{q}}^{2}=\frac{1}{\pi \Gamma(2 q-1)} \iint_{\mathbb{C}^{+}}|F(z)|^{2}[2 \operatorname{Re} z]^{2 q-2} d x d y, \quad z=x+i y,
$$

and for $q=\frac{1}{2}, K_{1 / 2}(w, z)$ is the Szegö reproducing kernel on the half plane $\mathbb{C}^{+}$ comprising all holomorphic functions $F(z)$ on $\mathbb{C}^{+}$with finite norms

$$
\|F\|_{\mathcal{D}_{1 / 2}}^{2}=\frac{1}{2 \pi} \sup _{x>0} \int_{\mathbb{R}}|F(x+i y)|^{2} d y .
$$

For $F \in \mathcal{D}_{q}, G \in \mathcal{D}_{p}$ such that 


$$
F(z)=\int_{0}^{\infty} e^{-z t} f(t) d t \quad \text { and } \quad G(z)=\int_{0}^{\infty} e^{-z t} g(t) d t
$$

we have the expression

$$
F(z) G(z)=\int_{0}^{\infty} e^{-z t}(f * g)(t) d t
$$

where

$$
(f * g)(t)=\int_{0}^{t} f(s) g(t-s) d s, \quad t>0
$$

It is easy to see that

$$
K_{q}(w, z) K_{p}(w, z)=\frac{\Gamma(2 q) \Gamma(2 p)}{\Gamma(2 p+2 q)} K_{q+p}(w, z) \quad \text { for } w, z \in \mathbb{C}^{+} .
$$

So, by using [1, Corollary 1$]$ (see also [6, 8], or [10]), we have the following inequality

$$
\|F G\|_{\mathcal{D}_{q+p}}^{2} \leq \frac{\Gamma(2 q) \Gamma(2 p)}{\Gamma(2 p+2 q)}\|F\|_{\mathcal{D}_{q}}^{2}\|G\|_{\mathcal{D}_{p}}^{2}
$$

for $F \in \mathcal{D}_{q}, G \in \mathcal{D}_{p}$. Furthermore, we have Theorem 4.1 whose proof can be done similarly to that of Theorem 2.1 .

Theorem 4.1. Let $p>0, q>0$ and $F_{j} \in \mathcal{D}_{q}, G_{j} \in \mathcal{D}_{p}$ for all $j=1,2, \ldots, m$. Then, we have the following inequality

$$
\begin{aligned}
& \operatorname{det}\left[\left\langle F_{i} G_{i}, F_{j} G_{j}\right\rangle_{\mathcal{D}_{q+p}}\right]_{i, j=1}^{m} \\
\leq & \left(\frac{\Gamma(2 q) \Gamma(2 p)}{\Gamma(2 p+2 q)}\right)^{m} \operatorname{det}\left[\left\langle F_{i}, F_{j}\right\rangle_{\mathcal{D}_{q}}\left\langle G_{i}, G_{j}\right\rangle_{\mathcal{D}_{p}}\right]_{i, j=1}^{m} .
\end{aligned}
$$

If $F_{j} \in \mathcal{D}_{q}$ and $G_{j} \in \mathcal{D}_{p}$ such that

$$
F_{j}(z)=A_{j} \frac{\Gamma(2 q)}{\left(\overline{w_{j}}+z\right)^{2 q}}, \quad G_{j}(z)=B_{j} \frac{\Gamma(2 p)}{\left(\overline{w_{j}}+z\right)^{2 p}}, \quad z \in \mathbb{C}^{+}
$$

for some $w_{j} \in \mathbb{C}^{+}$and some constants $A_{j}$ and $B_{j}, j=1,2, \ldots, m$, then the equality holds in (4.1).

Next, for a positive continuous function $\rho$ on $\mathbb{R}$, let us consider the kernel

$$
K_{\rho}(x, y)=\frac{1}{2 \pi} \int_{\mathbb{R}} e^{i \xi(y-x)} \rho(\xi) d \xi .
$$

Then, the images $F(x)$ of the transform

$$
F(x)=\frac{1}{2 \pi} \int_{\mathbb{R}} f(\xi) e^{-i \xi x} d \xi
$$


for functions $f$ satisfying

$$
\frac{1}{2 \pi} \int_{\mathbb{R}} \frac{|f(x)|^{2}}{\rho(x)} d x<\infty
$$

belong to the reproducing kernel Hilbert space $\mathcal{L}_{\rho}=\mathcal{H}_{K_{\rho}}$ admitting the reproducing kernel $K_{\rho}(x, y)$ and we have the isometrical identity

$$
\|F\|_{\mathcal{L}_{\rho}}^{2}=\frac{1}{2 \pi} \int_{\mathbb{R}} \frac{|f(x)|^{2}}{\rho(x)} d x .
$$

See [20, pp. 89-90].

Let $\rho_{j}, j=1,2$, be two positive continuous functions on $\mathbb{R}$ such that there exists

$$
\rho(x)=\left(\rho_{1} * \rho_{2}\right)(x):=\int_{\mathbb{R}} \rho_{1}(\xi) \rho_{2}(x-\xi) d \xi, \quad x \in \mathbb{R},
$$

and let $F \in \mathcal{L}_{\rho_{1}}$ and $G \in \mathcal{L}_{\rho_{2}}$ with

$$
F(x)=\frac{1}{2 \pi} \int_{\mathbb{R}} f(\xi) e^{-i \xi x} d \xi \quad \text { and } \quad G(x)=\frac{1}{2 \pi} \int_{\mathbb{R}} g(\xi) e^{-i \xi x} d \xi, \quad x \in \mathbb{R}
$$

for functions $f$ and $g$ satisfying

$$
\frac{1}{2 \pi} \int_{\mathbb{R}} \frac{|f(x)|^{2}}{\rho_{1}(x)} d x<\infty \quad \text { and } \quad \frac{1}{2 \pi} \int_{\mathbb{R}} \frac{|g(x)|^{2}}{\rho_{2}(x)} d x<\infty .
$$

Then,

$$
F(x) G(x)=\frac{1}{2 \pi} \int_{\mathbb{R}} \frac{1}{2 \pi}(f * g)(\xi) e^{-i \xi x} d \xi,
$$

and moreover, by using the following inequality (see [21, p. 121] or [1, Theorem 2])

$$
\int_{\mathbb{R}} \frac{|(f * g)(x)|^{2}}{\rho(x)} d x \leq \int_{\mathbb{R}} \frac{|f(x)|^{2}}{\rho_{1}(x)} d x \int_{\mathbb{R}} \frac{|g(x)|^{2}}{\rho_{2}(x)} d x
$$

we have

$$
\|F G\|_{\mathcal{L}_{\rho}}^{2} \leq \frac{1}{2 \pi}\|F\|_{\mathcal{L}_{\rho_{1}}}^{2}\|G\|_{\mathcal{L}_{\rho_{2}}}^{2}
$$

Moreover, we have the following theorem.

Theorem 4.2. Let $\rho_{j}, j=1,2$, be two positive continuous functions on $\mathbb{R}$ such that there exists

$$
\rho(x)=\left(\rho_{1} * \rho_{2}\right)(x):=\int_{\mathbb{R}} \rho_{1}(\xi) \rho_{2}(x-\xi) d \xi, \quad x \in \mathbb{R},
$$

and let $F_{j} \in \mathcal{L}_{\rho_{1}}, G_{j} \in \mathcal{L}_{\rho_{2}}$ for all $j=1,2, \ldots, m$. Then, we have the following inequality

$$
\operatorname{det}\left[\left\langle F_{i} G_{i}, F_{j} G_{j}\right\rangle_{\mathcal{L}_{\rho}}\right]_{i, j=1}^{m} \leq\left(\frac{1}{2 \pi}\right)^{m} \operatorname{det}\left[\left\langle F_{i}, F_{j}\right\rangle_{\mathcal{L}_{\rho_{1}}}\left\langle G_{i}, G_{j}\right\rangle_{\mathcal{L}_{\rho_{2}}}\right]_{i, j=1}^{m} .
$$




$$
\text { If } F_{j} \in \mathcal{L}_{\rho_{1}} \text { and } G_{j} \in \mathcal{L}_{\rho_{2}} \text { such that }
$$

$$
F_{j}(x)=\frac{A_{j}}{2 \pi} \int_{\mathbb{R}} e^{i \xi\left(y_{j}-x\right)} \rho_{1}(\xi) d \xi, \quad G_{j}(x)=\frac{B_{j}}{2 \pi} \int_{\mathbb{R}} e^{i \xi\left(y_{j}-x\right)} \rho_{2}(\xi) d \xi, \quad x \in \mathbb{C}
$$

for some $y_{j} \in \mathbb{C}$ and some constants $A_{j}$ and $B_{j}, j=1,2, \ldots, m$, then the equality holds in (4.4).

\section{Sobolev Hilbert Spaces}

First, for $a>0, b>0$ we examine the simplest Sobolev space $\mathcal{S}(a, b)$ on $\mathbb{R}$ consisting of all complex-valued and absolutely continuous functions $F(x)$ with finite norms

$$
\|F\|_{\mathcal{S}(a, b)}^{2}=\int_{\mathbb{R}}\left\{a^{2}\left|F^{\prime}(x)\right|^{2}+b^{2}|F(x)|^{2}\right\} d x<\infty .
$$

Note that (see [19])

$$
K_{a, b}(x, y)=\frac{1}{2 a b} e^{-\frac{b}{a}|x-y|}=\frac{1}{2 \pi} \int_{\mathbb{R}} \frac{e^{i \xi(x-y)}}{a^{2} \xi^{2}+b^{2}} d \xi
$$

is the reproducing kernel for the Sobolev Hilbert space $\mathcal{S}(a, b)$. Hence, any member $F \in \mathcal{S}(a, b)$ is expressible in the form

$$
F(x)=\frac{1}{2 \pi} \int_{\mathbb{R}} f(\xi) e^{i \xi x} d \xi
$$

for a complex-valued function $f$ satisfying

$$
\int_{\mathbb{R}} \frac{|f(x)|^{2}}{a^{2} x^{2}+b^{2}} d x<\infty
$$

and we have the isometrical identity

$$
\|F\|_{\mathcal{S}(a, b)}^{2}=\frac{1}{2 \pi} \int_{\mathbb{R}} \frac{|f(x)|^{2}}{a^{2} x^{2}+b^{2}} d x .
$$

Let $a_{1}, a_{2}, b_{1}, b_{2}$ be positive real numbers and $a=a_{1} a_{2}, b=\left(a_{1} b_{2}+a_{2} b_{1}\right)$. Then,

$$
K_{a_{1}, b_{1}}(x, y) K_{a_{2}, b_{2}}(x, y)=\frac{1}{2}\left(\frac{a_{1}}{b_{1}}+\frac{a_{2}}{b_{2}}\right) K_{a, b}(x, y) \quad \text { for } x, y \in \mathbb{R} .
$$

Hence, for $F \in \mathcal{S}\left(a_{1}, b_{1}\right)$ and $G \in \mathcal{S}\left(a_{2}, b_{2}\right)$ we have $F G \in \mathcal{S}(a, b)$, and moreover (see [19, Theorem 1.1]),

$$
\|F G\|_{\mathcal{S}(a, b)}^{2} \leq \frac{1}{2}\left(\frac{a_{1}}{b_{1}}+\frac{a_{2}}{b_{2}}\right)\|F\|_{\mathcal{S}\left(a_{1}, b_{1}\right)}^{2}\|G\|_{\mathcal{S}\left(a_{2}, b_{2}\right)}^{2}
$$

So, in view of Theorem 4.2, we get the following theorem. 
Theorem 5.1. Let $a_{1}, a_{2}, b_{1}$ and $b_{2}$ be positive real numbers and set $a=a_{1} a_{2}, b=$ $\left(a_{1} b_{2}+a_{2} b_{1}\right)$. Then, the following inequality

$$
\begin{aligned}
& \operatorname{det}\left[\left\langle F_{i} G_{i}, F_{j} G_{j}\right\rangle_{\mathcal{S}(a, b)}\right]_{i, j=1}^{m} \\
& \leq\left[\frac{1}{2}\left(\frac{a_{1}}{b_{1}}+\frac{a_{2}}{b_{2}}\right)\right]^{m} \operatorname{det}\left[\left\langle F_{i}, F_{j}\right\rangle_{\mathcal{S}\left(a_{1}, b_{1}\right)}\left\langle G_{i}, G_{j}\right\rangle_{\mathcal{S}\left(a_{2}, b_{2}\right)}\right]_{i, j=1}^{m} .
\end{aligned}
$$

holds for $F_{j} \in \mathcal{S}\left(a_{1}, b_{1}\right)$ and $G_{j} \in \mathcal{S}\left(a_{2}, b_{2}\right)$ for $j=1,2, \ldots, m$.

If $F_{j} \in \mathcal{S}\left(a_{1}, b_{1}\right)$ and $G_{j} \in \mathcal{S}\left(a_{2}, b_{2}\right)$ such that

$$
F_{j}(x)=\frac{A_{j}}{2 a_{1} b_{1}} e^{-\frac{b_{1}}{a_{1}}\left|x-y_{j}\right|}, \quad G_{j}(x)=\frac{B_{j}}{2 a_{2} b_{2}} e^{-\frac{b_{2}}{a_{2}}\left|x-y_{j}\right|}, \quad x \in \mathbb{R}
$$

for some $y_{j} \in \mathbb{R}$ and some constants $A_{j}$ and $B_{j}, j=1,2, \ldots, m$, then the equality holds in (5.2).

Finally, let $\Omega=(a, b)(-\infty \leq a<b \leq \infty)$ be a finite or infinite interval of the real axis $\mathbb{R}=(-\infty, \infty)$. For a positive continuous function $\rho$ on $\Omega$, let $\mathcal{W}_{\rho}$ be the space of all functions $F$ which are complex-valued and absolutely continuous on $\Omega$ such that $\lim _{x \rightarrow a} F(x)=0$ and

$$
\int_{\Omega} \frac{\left|F^{\prime}(x)\right|^{2}}{\rho(x)} d x<\infty
$$

We note that (see [21, pp. 55-56] or [18]) $\mathcal{W}_{\rho}$ is a weighted Sobolev space admitting the reproducing kernel

with the norm

$$
K(x, s)=\int_{a}^{\min (x, s)} \rho(t) d t
$$

$$
\|F\|_{\mathcal{W}}^{2} \mathcal{W}_{\rho}=\int_{\Omega} \frac{\left|F^{\prime}(x)\right|^{2}}{\rho(x)} d x .
$$

Theorem 5.2. For two positive continuous functions $\rho_{1}$ and $\rho_{2}$ let us consider a new positive continuous function

$$
\rho(x)=\left(\int_{a}^{x} \rho_{1}(t) d t \int_{a}^{x} \rho_{2}(t) d t\right)^{\prime}, \quad x \in \Omega .
$$

Then, for $F_{j} \in \mathcal{W}_{\rho_{1}}, G_{j} \in \mathcal{W}_{\rho_{2}}, j=1,2, \ldots, m$, we have $F_{j} G_{j} \in \mathcal{W}_{\rho}$ and moreover,

$$
\operatorname{det}\left[\left\langle F_{i} G_{i}, F_{j} G_{j}\right\rangle_{\mathcal{W}_{\rho}}\right]_{i, j=1}^{m} \leq \operatorname{det}\left[\left\langle F_{i}, F_{j}\right\rangle_{\mathcal{W}_{\rho_{1}}}\left\langle G_{i}, G_{j}\right\rangle_{\mathcal{W}_{\rho_{2}}}\right]_{i, j=1}^{m} .
$$

If $F_{j} \in \mathcal{W}_{\rho_{1}}$ and $G_{j} \in \mathcal{W}_{\rho_{2}}$ such that

$$
F_{j}(x)=A_{j} \int_{a}^{\min \left(x, s_{j}\right)} \rho_{1}(t) d t, \quad G_{j}(x)=\int_{a}^{\min \left(x, s_{j}\right)} \rho_{2}(t) d t, \quad x \in \Omega
$$

for some $s_{j} \in \Omega$ and some constants $A_{j}$ and $B_{j}, j=1,2, \ldots, m$, then the equality holds in (5.5). 
Proof. Let $F_{j} \in \mathcal{W}_{\rho_{1}}, G_{j} \in \mathcal{W}_{\rho_{2}}, j=1,2, \ldots, m$. Then, from [9, Theorem 1.6] we see that $F_{j} G_{j} \in \mathcal{W}_{\rho}$. Since $F_{j}$ and $G_{j}$ are absolutely continuous with $\lim _{x \rightarrow a} F_{j}(x)=$ $0, \lim _{x \rightarrow a} G_{j}(x)=0$, then

$$
F_{j}(x)=\int_{a}^{x} F_{j}^{\prime}(t) d t \quad \text { and } \quad G_{j}(x)=\int_{a}^{x} G_{j}^{\prime}(t) d t, \quad x \in \Omega,
$$

for all $j=1,2, \ldots, m$. So, we have

$$
\begin{aligned}
& \operatorname{det}\left[\left(F_{i} G_{i}\right)^{\prime}\left(x_{j}\right)\right]_{i, j=1}^{m} \\
= & \operatorname{det}\left[F_{i}^{\prime}\left(x_{j}\right) G_{i}\left(x_{j}\right)+F_{i}\left(x_{j}\right) G_{i}^{\prime}\left(x_{j}\right)\right]_{i, j=1}^{m} \\
= & \operatorname{det}\left[F_{i}^{\prime}\left(x_{j}\right) \int_{a}^{x_{j}} G_{i}^{\prime}\left(t_{j}\right) d t_{j}+\int_{a}^{x_{j}} F_{i}^{\prime}\left(t_{j}\right) d t_{j} G_{i}^{\prime}\left(x_{j}\right)\right]_{i, j=1}^{m} \\
= & \int_{a}^{x_{1}} \cdots \int_{a}^{x_{m}} \sum_{\substack{\left\{\alpha_{k}, \beta_{k}\right\}=\left\{x_{k}, t_{k}\right\} \\
k=1,2, \ldots, m}} \operatorname{det}\left[F_{i}^{\prime}\left(\alpha_{j}\right) G_{i}^{\prime}\left(\beta_{j}\right)\right]_{i, j=1}^{m} d t_{1} \cdots d t_{m} .
\end{aligned}
$$

By using the Cauchy-Schwarz inequality, we get

$$
\begin{aligned}
& \left|\operatorname{det}\left[\left(F_{i} G_{i}\right)^{\prime}\left(x_{j}\right)\right]_{i, j=1}^{m}\right|^{2} \\
& \leq \int_{a}^{x_{1}} \cdots \int_{a}^{x_{m}} \sum_{\substack{\left\{\alpha_{k}, \beta_{k}\right\}=\left\{x_{k}, t_{k}\right\} \\
k=1,2, \ldots, m}} \frac{\left|\operatorname{det}\left[F_{i}^{\prime}\left(\alpha_{j}\right) G_{i}^{\prime}\left(\beta_{j}\right)\right]_{i, j=1}^{m}\right|^{2}}{\prod_{j=1}^{m} \rho_{1}\left(\alpha_{j}\right) \rho_{2}\left(\beta_{j}\right)} d t_{1} \cdots d t_{m} \\
& \quad \times \int_{a}^{x_{1}} \cdots \int_{a}^{x_{m}} \sum_{\substack{\left\{\alpha_{k}, \beta_{k}\right\}=\left\{x_{k}, t_{k}\right\} \\
k=1,2, \ldots, m}}^{m} \prod_{j=1}^{m} \rho_{1}\left(\alpha_{j}\right) \rho_{2}\left(\beta_{j}\right) d t_{1} \cdots d t_{m} .
\end{aligned}
$$

Note that,

$$
\sum_{\substack{\left\{\alpha_{k}, \beta_{k}\right\}=\left\{x_{k}, t_{k}\right\} \\ k=1,2, \ldots, m}} \prod_{j=1}^{m} \rho_{1}\left(\alpha_{j}\right) \rho_{2}\left(\beta_{j}\right)=\prod_{j=1}^{m}\left(\rho_{1}\left(x_{j}\right) \rho_{2}\left(t_{j}\right)+\rho_{1}\left(t_{j}\right) \rho_{2}\left(x_{j}\right)\right),
$$

and

$$
\begin{aligned}
& \sum_{\substack{\left\{\alpha_{k}, \beta_{k}\right\}=\left\{x_{k}, t_{k}\right\} \\
k=1,2, \ldots, m}} \frac{\left|\operatorname{det}\left[F_{i}^{\prime}\left(\alpha_{j}\right) G_{i}^{\prime}\left(\beta_{j}\right)\right]_{i, j=1}^{m}\right|^{2}}{\prod_{j=1}^{m} \rho_{1}\left(\alpha_{j}\right) \rho_{2}\left(\beta_{j}\right)} \\
& =\sum_{\substack{\left\{\alpha_{k}, \beta_{k}\right\}=\left\{x_{k}, t_{k}\right\} \\
k=1,2, \ldots, m}} \sum_{\sigma \in S_{m}} \sum_{\gamma \in S_{m}} \operatorname{sgn} \sigma \operatorname{sgn} \gamma \prod_{i=1}^{m} \frac{F_{i}^{\prime}\left(\alpha_{\sigma(i)}\right) \overline{F_{i}^{\prime}\left(\alpha_{\gamma(i)}\right)} G_{i}^{\prime}\left(\beta_{\sigma(i)}\right) \overline{G_{i}^{\prime}\left(\beta_{\gamma(i)}\right)}}{\rho_{1}\left(\alpha_{i}\right) \rho_{2}\left(\beta_{i}\right)} \\
& =\sum_{\substack{\left\{\alpha_{k}, \beta_{k}\right\}=\left\{x_{k}, t_{k}\right\} \\
k=1,2, \ldots, m}} \sum_{\sigma \in S_{m}} \operatorname{det}\left[\frac{F_{i}^{\prime}\left(\alpha_{\sigma(i)}\right) \overline{F_{j}^{\prime}\left(\alpha_{\sigma(i)}\right)} G_{i}^{\prime}\left(\beta_{\sigma(i)}\right) \overline{G_{j}^{\prime}\left(\beta_{\sigma(i)}\right)}}{\left.\rho_{1}\left(\alpha_{\sigma(i)}\right)\right) \rho_{2}\left(\beta_{\sigma(i)}\right)}\right]_{i, j=1}^{m}
\end{aligned}
$$




$$
\begin{aligned}
= & \sum_{\sigma \in S_{m}} \operatorname{det}\left[\frac{F_{i}^{\prime}\left(x_{\sigma(i)}\right) \overline{F_{j}^{\prime}\left(x_{\sigma(i)}\right)} G_{i}^{\prime}\left(t_{\sigma(i)}\right) \overline{G_{j}^{\prime}\left(t_{\sigma(i)}\right)}}{\left.\rho_{1}\left(x_{\sigma(i)}\right)\right) \rho_{2}\left(t_{\sigma(i)}\right)}\right. \\
& \left.+\frac{F_{i}^{\prime}\left(t_{\sigma(i)}\right) \overline{F_{j}^{\prime}\left(t_{\sigma(i)}\right)} G_{i}^{\prime}\left(x_{\sigma(i)}\right) \overline{G_{j}^{\prime}\left(x_{\sigma(i)}\right)}}{\left.\rho_{1}\left(t_{\sigma(i)}\right)\right) \rho_{2}\left(x_{\sigma(i)}\right)}\right]_{i, j=1}^{m} .
\end{aligned}
$$

So, we have

$$
\int_{a}^{x_{1}} \cdots \int_{a}^{x_{m}} \sum_{\substack{\left\{\alpha_{k}, \beta_{k}\right\}=\left\{x_{k}, t_{k}\right\} \\ k=1,2, \ldots, m}} \prod_{j=1}^{m} \rho_{1}\left(\alpha_{j}\right) \rho_{2}\left(\beta_{j}\right) d t_{1} \cdots d t_{m}=\prod_{j=1}^{m} \rho\left(x_{j}\right),
$$

and

$$
\begin{aligned}
& \int_{a}^{x_{1}} \cdots \int_{a}^{x_{m}} \sum_{\substack{\left\{\alpha_{k}, \beta_{k}\right\}=\left\{x_{k}, t_{k}\right\} \\
k=1,2, \ldots, m}} \frac{\left|\operatorname{det}\left[F_{i}^{\prime}\left(\alpha_{j}\right) G_{i}^{\prime}\left(\beta_{j}\right)\right]_{i, j=1}^{m}\right|^{2}}{\prod_{j=1}^{m} \rho_{1}\left(\alpha_{j}\right) \rho_{2}\left(\beta_{j}\right)} d t_{1} \cdots d t_{m} \\
& =\sum_{\sigma \in S_{m}} \operatorname{det}\left[\left(\int_{a}^{x_{\sigma(i)}} \frac{F_{i}^{\prime}\left(t_{\sigma(i)}\right) \overline{F_{j}^{\prime}\left(t_{\sigma(i)}\right)}}{\rho_{1}\left(t_{\sigma(i)}\right)} d t_{\sigma(i)} \int_{a}^{x_{\sigma(i)}} \frac{G_{i}^{\prime}\left(t_{\sigma(i)}\right) \overline{G_{j}^{\prime}\left(t_{\sigma(i)}\right)}}{\rho_{2}\left(t_{\sigma(i)}\right)} d t_{\sigma(i)}\right)^{\prime}\right]_{i, j=1}^{m}
\end{aligned}
$$

Therefore,

$$
\begin{aligned}
\frac{\left|\operatorname{det}\left[\left(F_{i} G_{i}\right)^{\prime}\left(x_{j}\right)\right]_{i, j=1}^{m}\right|^{2}}{\prod_{j=1}^{m} \rho\left(x_{j}\right)} \leq \sum_{\sigma \in S_{m}} \operatorname{det}[ & \left(\int_{a}^{x_{\sigma(i)}} \frac{F_{i}^{\prime}\left(t_{\sigma(i)}\right) \overline{F_{j}^{\prime}\left(t_{\sigma(i)}\right)}}{\rho_{1}\left(t_{\sigma(i)}\right)} d t_{\sigma(i)}\right. \\
& \left.\left.\int_{a}^{x_{\sigma(i)}} \frac{G_{i}^{\prime}\left(t_{\sigma(i)}\right) \overline{G_{j}^{\prime}\left(t_{\sigma(i)}\right)}}{\rho_{2}\left(t_{\sigma(i)}\right)} d t_{\sigma(i)}\right)^{\prime}\right]_{i, j=1}^{m}
\end{aligned}
$$

which yields (5.5).

\section{ACKNOWLEDGMENTS}

The results in the paper benefited from numerous communications with Professor Saburou Saitoh over the years in writing. So, the authors wish to express our deep thanks to him. Our thanks also go out to the anonymous referee for his/her careful reading and valuable comments.

\section{REFERENCES}

1. K. F. Andersen, Weighted inequalities for iterated convolutions, Proc. Amer. Math. Soc., 127 (1999), 2643-2651. 
2. N. Aronszajn, Theory of reproducing kernels, Trans. Amer. Math. Soc., 68 (1950), 337404.

3. J. Burbea, Total positivity of certain reproducing kernels, Pacific J. Math., 67 (1976), 101-130.

4. J. Burbea, A Dirichlet norm inequality and some inequalities for reproducing kernel spaces, Proc. Amer. Math. Soc., 83 (1981), 279-285.

5. J. Burbea, Norm inequalities of exponential type for holomorphic functions, Kodai Math. J., 5 (1982), 339-354.

6. J. Burbea, Inequalities for weighted $L^{2}$-functions on the half-line, Arch. Math., 47 (1986), 427-437.

7. L. P. Castro and S. Saitoh, New convolutions and norm inequalities, Math. Inequal. Appl. 15 (2012), 107-716.

8. M. Cwikel and R. Kerman, On a convolution inequality of Saitoh, Proc. Amer. Math. Soc., 124 (1996), 773-777.

9. D. T. Duc and N. D. V. Nhan, Generalize some norm inequalities of Saitoh, Kodai Math. J., 34 (2011), 191-201.

10. N. D. V. Nhan and D. T. Duc, Fundamental inequalities for the iterated Laplace convolution in weighted $L_{p}$ spaces and their applications, Integr. Transform. and Special Funct., 19 (2008), 655-664.

11. N. D. V. Nhan and D. T. Duc, Convolution inequalities in $l_{p}$ weighted spaces, Canad. Math. Bull., 55 (2012), 355-367.

12. N. D. V. Nhan and D. T. Duc, Norm inequalities for Mellin convolutions and their applications, Complex Analysis and Operator Theory, 2012, DOI: 10.1007/s11785-0120224-z.

13. N. D. V. Nhan, D. T. Duc and V. K. Tuan, Weighted norm inequalities for a nonlinear transform, Comp. Math. Appl., 61 (2011) 832-839.

14. S. Saitoh, Some inequalities for analytic functions with a finite Dirichlet integral on the unit disc, Math. Ann., 246 (1979), 69-77.

15. S. Saitoh, The Bergman norm and the Szegö norm, Trans. Amer. Math. Soc., 249 (1979), 261-279.

16. S. Saitoh, Some inequalities for entire function, Proc. Amer. Math. Soc., 80 (1980), 254-258.

17. S. Saitoh, A fundamental inequality in the convolution of $L_{2}$ functions on the half line, Proc. Amer. Math. Soc., 91 (1984), 285-286.

18. S. Saitoh, Hilbert spaces admitting reproducing kernels on the real line and related fundamental inequalities, Riazi, J. Kar. Math. Assoc., 6 (1984), 25-31.

19. S. Saitoh, Inequalities in the most simple Sobolev space and convolutions of $L_{2}$ functions with weights, Proc. Amer. Math. Soc., 118 (1993), 515-520. 
20. S. Saitoh, Integral Transforms, Reproducing Kernels and Their Applications, Pitman Research Notes in Mathematics Series, 369, Addison Wesley Longman, UK, 1997.

21. S. Saitoh, Various operators in Hilbert space introduced by transforms, International Journal of Applied Mathematics, 1 (1999), 111-126.

22. A. Yamada, Equality conditions for general norm inequalities in reproducing kernel Hilbert spaces, Advances in Analysis, World Scientific, 2005, pp. 447-455.

Nguyen Du Vi Nhan and Dinh Thanh Duc

Department of Mathematics

Quy Nhon University

Binh Dinh

Vietnam

E-mail: ndvynhan@gmail.com

nguyenduvinhan@qnu.edu.vn

ducdinh2002@yahoo.com

dinhthanhduc@qnu.edu.vn 